\title{
ON NONDENSELY DEFINED SEMILINEAR STOCHASTIC FUNCTIONAL DIFFERENTIAL EQUATIONS WITH NONLOCAL CONDITIONS
}

\author{
M. BENCHOHRA, S. K. NTOUYAS, AND A. OUAHAB
}

Received 9 October 2004; Revised 5 March 2005; Accepted 7 March 2005

The nonlinear alternative of Leray-Schauder type is used to investigate the existence of solutions for first-order semilinear stochastic functional differential equations in Hilbert spaces.

Copyright (c) 2006 M. Benchohra et al. This is an open access article distributed under the Creative Commons Attribution License, which permits unrestricted use, distribution, and reproduction in any medium, provided the original work is properly cited.

\section{Introduction}

This paper is concerned with the existence of integral solutions for initial value problems for first-order stochastic semilinear functional differential equations with nonlocal conditions in Hilbert spaces. More precisely in Section 3, we consider first-order stochastic semilinear functional differential equations of the form

$$
\begin{gathered}
y^{\prime}(t)=A y(t)+f\left(t, y_{t}\right) \frac{d w(t)}{d t}, \quad t \in J:=[0, b], \\
y(t)+h_{t}(y)=\phi(t), \quad t \in[-r, 0],
\end{gathered}
$$

where $f: J \times \widehat{M}_{2}([-r, 0], H) \rightarrow H$ is a given function, $A: D(A) \subset H \rightarrow H$ is a nondensely defined closed linear operator on $H$, the function $w(t)$ is a Hilbert space Q-valued Wiener process, $\phi \in \widehat{M}_{2}([-r, 0], D(A)), 0<r<\infty$, is a suitable initial random function independent of $w(t), h: \widehat{M}_{2}([-r, 0], D(A)) \rightarrow D(A), H$ a real separable Hilbert space with inner product $\langle\cdot, \cdot\rangle$ and norm $|\cdot|$, and $\widehat{M}_{2}$ is a class of $H$-valued stochastic processes that will be specified later (see Section 2 ). Here $y_{t}(\cdot)$ represents the history of stochastic processes state from time $t-r$, up to the present time $t$. The nonlocal conditions were initiated by Byszewski. We refer the readers to [4] and the references cited therein for motivation regarding the nonlocal initial conditions. The nonlocal condition can be applied in physics 
with better effect than the classical initial condition $y(0)=y_{0}$. For example, $h_{t}(y)$ may be given by

$$
h_{t}(y)=\sum_{i=1}^{p} c_{i} y\left(t_{i}+t\right), \quad t \in[-r, 0],
$$

where $c_{i}, i=1, \ldots, p$, are given constants and $0<t_{1}<\cdots<t_{p} \leq b$. At time $t=0$, we have

$$
h_{0}(y)=\sum_{i=1}^{p} c_{i} y\left(t_{i}\right)
$$

Random differential and integral equations play an important role in characterizing many social, physical, biological, and engineering problems; see, for instance, the monographs of Da Prato and Zabczyk [6] and Sobczyk [14]. For example, a stochastic model for drug distribution in a biological system was described by Tsokos and Padgett [16] to be a closed system with a simplified heat, one organ or capillary bed, and recirculation of blood with a constant rate of flow, where the heart is considered as a mixing chamber of constant volume. The basic theory concerning stochastic differential equations can be found in the monographs of Bharucha-Reid [3], Da Prato and Zabczyk [6], and Tsokos and Padgett [16]. For recent results, we refer to the papers of Liu [11], McKibben [12, 13], and Taniguchi [15].

Recently, Balasubramaniam and Ntouyas [2] studied the semilinear stochastic evolution delay equations with nonlocal conditions, where $A$ is a densely defined linear operator. Our goal here is to extend the results of Balasubramaniam and Ntouyas [2], where $A$ is nondensely defined. These results can be seen as a contribution to the literature.

\section{Preliminaries}

In this section, we introduce notations, definitions, and preliminary facts which are used throughout this paper.

Let $K$ be another real separable Hilbert space and let $w(t), t \geq 0$, be a $K$-valued Wiener process with mean zero and covariance operator $Q$ with $\operatorname{tr} Q<\infty(\operatorname{tr} Q$ denotes the trace of the operator $Q$ ) defined by

$$
E\langle w(t), g\rangle\langle w(s), h\rangle=(t \wedge s)\langle Q g, h\rangle \quad \text { for every } g, h \in K
$$

where $\langle\cdot, \cdot\rangle$ denotes the inner product and $E$ stands for integration with respect to probability measure $P$. Let $L(K, H)$ denote the space of bounded linear operators from $K$ into $H$. For $g_{1}, g_{2} \in L(K, H)$, we define $\left\langle\left\langle g_{1}, g_{2}\right\rangle\right\rangle=\operatorname{tr}\left(g_{1} Q g_{2}^{*}\right)$, where $g_{2}^{*}$ is the adjoint of the operator $g_{2}$ and $Q$ is the nuclear operator associated with the Brownian motion, where $Q \in L_{n}^{+}(K)$, the space of positive nuclear operator in $K$. Let $L\left(K_{Q}, H\right)$ denote the completion of $L(K, H)$ with respect to the topology induced by the norm $\|\cdot\|_{2}$, where $\|g\|_{2}^{2}=$ $\langle\langle g, g\rangle\rangle$. Let $\left(\Omega, \mathscr{F}, \mathscr{F}_{t}, P, H\right)$ be a complete probability space furnished with a complete 
family of right continuous increasing $\sigma$-algebras $\left\{\mathscr{F}_{t}, t \in[0, T]\right\}$ satisfying $\mathscr{F}_{t} \subset \mathscr{F}_{\text {. }}$ Let $L^{2}\left(\Omega, \mathscr{F}_{F}, \mathscr{F}_{t}, P, H\right)$ be a space of all square random variables with values in $H$ that are measurable with respect to $\left\{\mathscr{F}_{t}, t \in[0, b]\right\}$. Let $\widehat{M}_{2}([-r, b], H)$ denote the classes of $H$-valued stochastic processes $\{\xi(t): t \in[-r, b]\}$ which are $\mathscr{F}_{t}$-adapted and have finite second moments, that is,

$$
\|\xi\|_{\widehat{M}_{2}}=\sup _{t \in[-r, b]}\left(E|\xi(t)|^{2}\right)^{1 / 2}<\infty
$$

It is easy to verify that $\widehat{M}_{2}$, furnished with the norm topology as defined above, is a Banach space. White noise is usually regarded as informal time derivative $w^{\prime}(t)$ of Brownian motion or Wiener process $w(t)$. In the Itô theory of stochastic integration, an integral with respect to $w^{\prime}(t)$ is rewritten as one with respect to $d w(t)$, that is,

$$
\int_{a}^{b} \psi(t) d w(t)=\int_{a}^{b} \psi(t) w^{\prime}(t) d t .
$$

The Itô integral $\int_{a}^{b} \psi(t) d w(t)$ is defined for any process $\psi(t)$ which satisfies the following conditions:

(1) $\psi$ is nonanticipating,

(2) almost all sample paths of $\psi$ belong to $L^{2}([a, b])$. Moreover, $\int_{a}^{b} \psi(t) d w(t) \in L^{2}(\Omega)$ if and only if $\psi \in L^{2}([a, b] \times \Omega)$. In fact the following equality holds:

$$
E\left|\int_{a}^{b} \psi(t) d w(t)\right|^{2}=E \int_{a}^{b}|\psi(t)|^{2} d t .
$$

For more details on Brownian motion and white noise, we refer the reader to the books of Hida [8] and Hida et al. [9].

$B(H)$ denotes the Banach space of bounded linear operators from $H$ into $H$ with norm

$$
\|N\|_{B(H)}=\sup \{|N(y)|:|y|=1\} .
$$

Definition 2.1 (see [1]). Let $E$ be a Banach space. An integrated semigroup is a family of operators $(S(t))_{t \geq 0}$ of bounded linear operators $S(t)$ on $E$ with the following properties:

(i) $S(0)=0$;

(ii) $t \rightarrow S(t)$ is strongly continuous;

(iii) $S(s) S(t)=\int_{0}^{s}(S(t+r)-S(r)) d r$, for all $t, s \geq 0$.

Definition 2.2 (see [10]). An operator $A$ is called a generator of an integrated semigroup if there exists $\omega \in \mathbb{R}$ such that $(\omega, \infty) \subset \rho(A)(\rho(A)$ is the resolvent set of $A)$ and there exists a strongly continuous exponentially bounded family $(S(t))_{t \geq 0}$ of bounded operators such that $S(0)=0$ and $R(\lambda, A):=(\lambda I-A)^{-1}=\lambda \int_{0}^{\infty} e^{-\lambda t} S(t) d t$ exists for all $\lambda$ with $\lambda>\omega$.

Proposition 2.3 (see [1]). Let $A$ be the generator of an integrated semigroup $(S(t))_{t \geq 0}$. Then for all $x \in E$ and $t \geq 0$,

$$
\int_{0}^{t} S(s) x d s \in D(A), \quad S(t) x=A \int_{0}^{t} S(s) x d s+t x .
$$




\section{Stochastic functional differential equations}

Definition 2.4 (see [10]). (i) An integrated semigroup $(S(t))_{t \geq 0}$ is called locally Lipschitz continuous if, for all $\tau>0$, there exists a constant $L$ such that

$$
|S(t)-S(s)| \leq L|t-s|, \quad t, s \in[0, \tau] .
$$

(ii) An integrated semigroup $(S(t))_{t \geq 0}$ is called nondegenerate if $S(t) x=0$, for all $t \geq 0$, implies that $x=0$.

Definition 2.5. We say that the linear operator $A$ satisfies the Hille-Yosida condition if there exist $M \geq 0$ and $\omega \in \mathbb{R}$ such that $(\omega, \infty) \subset \rho(A)$ and

$$
\sup \left\{(\lambda-\omega)^{n}\left|(\lambda I-A)^{-n}\right|: n \in \mathbb{N}, \lambda>\omega\right\} \leq M .
$$

Theorem 2.6 (see [10]). The following assertions are equivalent:

(H0) $A$ is the generator of a nondegenerate, locally Lipschitz continuous integrated semigroup;

(H1) A satisfies the Hille-Yosida condition.

If $A$ is the generator of an integrated semigroup $(S(t))_{t \geq 0}$ which is locally Lipschitz, then from [1], $S(\cdot) x$ is continuously differentiable if and only if $x \in \overline{D(A)}$ and $\left(S^{\prime}(t)\right)_{t \geq 0}$ is a $C_{0}$ semigroup on $\overline{D(A)}$.

Definition 2.7. A map $f: J \times \widehat{M}_{2}([-r, 0], H) \rightarrow H$ is said to be $L^{2}$-Carathéodory if

(i) $t \mapsto f(t, u)$ is measurable for each $u \in \widehat{M}_{2}([-r, 0], H)$;

(ii) $u \mapsto f(t, u)$ is continuous for almost all $t \in J$;

(iii) for each $q>0$, there exists $h_{q} \in L^{1}\left(J, \mathbb{R}_{+}\right)$such that

$$
|f(t, u)|^{2} \leq h_{q}(t) \quad \forall\|u\|_{\widehat{M}_{2}}^{2} \leq q \text { and for almost all } t \in J .
$$

In what follows, we will assume that $f$ is an $L^{2}$-Carathéodory function.

\section{Main result}

The aim of this section is to study the existence of integral solutions for the nonlocal problem (1.1).

Definition 3.1. For any $H$-valued $\mathscr{F}_{0}$-measurable stochastic processes $\phi$ satisfying the condition $E\|\phi(t)\|^{2}<\infty$ for every $t \in[-r, 0]$, an element $y \in \widehat{M}_{2}$ is said to be an integral solution of (1.1) if

(i) $y(t)+h_{t}(y)=\phi(t), t \in[-r, 0]$,

(ii) $\int_{0}^{t} y(s) d s \in D(A), t \in J$,

(iii) $y(t)=S^{\prime}(t)\left[\phi(0)-h_{0}(y)\right]+A \int_{0}^{t} y(s) d s+\int_{0}^{t} f\left(s, y_{s}\right) d w(s), t \in J$.

From the definition it follows that $y(t) \in \overline{D(A)}, t \geq 0$. Moreover, $y$ satisfies the following variation of constant formula:

$$
y(t)=S^{\prime}(t)\left[\phi(0)-h_{t}(y)\right]+\frac{d}{d t} \int_{0}^{t} S(t-s) f\left(s, y_{s}\right) d w(s), \quad t \in J .
$$

We are now in a position to state and prove our existence result for the problem (1.1). 
Theorem 3.2. Assume (H1) and

(H2) $w$ is an $H$-valued Wiener process defined on Hilbert space $K$;

(H3) $S^{\prime}(t), t>0$, is compact and there exist $M>0, \omega \in \mathbb{R}$ such that

$$
\left\|S^{\prime}(t)\right\|_{B(H)}^{2} \leq M e^{\omega t}, \quad t \geq 0
$$

(H4) the function $h$ is continuous with respect to $t$ and there exists a constant $\beta>0$ such that

$$
\left|h_{t}(u)\right|^{2} \leq \beta, \quad u \in \widehat{M}_{2}([-r, 0], H),
$$

and for each $k>0$, the set

$$
\left\{\phi(0)-h_{0}(y): y \in \widehat{M}_{2}([-r, 0], H),\|y\|_{\widehat{M}_{2}} \leq k\right\}
$$

is precompact in $H$;

(H5) there exist a continuous nondecreasing function $\psi:[0, \infty) \rightarrow(0, \infty)$ and $p \in L^{1}([0, b]$, $\mathbb{R}_{+}$) such that

$E|f(t, u)|^{2} \leq p(t) \psi\left(E\|u\|_{\widehat{M}_{2}}^{2}\right) \quad$ for a.e. $t \in[0, b]$ and each $u \in \widehat{M}_{2}([-r, 0], \overline{D(A)})$,

$$
\int_{0}^{b} p_{*}(s) d s<\int_{c}^{\infty} \frac{d x}{x+\psi(x)}
$$

where $p_{*}(t)=\max (|\omega|, 2 M p(t))$ and $c=4 M E\left(|\phi(0)|^{2}+\beta\right)$ are satisfied. Then the problem (1.1) has at least one integral solution on $[-r, b]$.

Proof. We transform the problem (1.1) into a fixed-point problem. Consider the operator $N: \widehat{M}_{2}([-r, b], \overline{D(A)}) \rightarrow \widehat{M}_{2}([-r, b], \overline{D(A)})$ defined by

$$
N(y)(t):= \begin{cases}\phi(t)-h_{t}(y) & \text { if } t \in[-r, 0], \\ S^{\prime}(t)\left[\phi(0)-h_{0}(y)\right]+\frac{d}{d t} \int_{0}^{t} S(t-s) f\left(s, y_{s}\right) d w(s) & \text { if } t \in[0, b] .\end{cases}
$$

Remark 3.3. It is clear that the fixed points of $N$ are integral solutions to (1.1).

In order to use the Leray-Schauder alternative, we will obtain a priori estimates for the solutions of the integral equation

$$
y(t)=\lambda\left[S^{\prime}(t)\left[\phi(t)-h_{0}(y)\right]+\frac{d}{d t} \int_{0}^{t} S(t-s) f\left(s, y_{s}\right) d s\right]
$$

and $y(t)=\lambda\left[\phi(t)-h_{t}(y)\right], t \in[-r, 0], \lambda \in(0,1)$. Hence

$$
\begin{aligned}
|y(t)|^{2} & =\lambda^{2}\left|S^{\prime}(t)\left[\phi(0)-h_{0}(y)\right]+\frac{d}{d t} \int_{0}^{t} S(t-s) f\left(s, y_{s}\right) d w(s)\right|^{2} \\
& \leq 2\left|S^{\prime}(t)\left[\phi(0)-h_{0}(y)\right]\right|^{2}+2\left|\frac{d}{d t} \int_{0}^{t} S(t-s) f\left(s, y_{s}\right) d w(s)\right|^{2} .
\end{aligned}
$$


6 Stochastic functional differential equations

Thus by (H3), (H4), and (H5), we have

$$
E\left(|y(t)|^{2}\right) \leq 4 M e^{\omega t} E\left(|\phi(0)|^{2}+\beta\right)+2 M e^{\omega t} \int_{0}^{t} e^{-\omega s} p(s) \psi\left(E\left(\left\|y_{s}\right\|^{2}\right)\right) d s
$$

We consider the function $\mu$ defined by

$$
\mu(t)=\sup \{|y(s)|: 0 \leq s \leq t\}, \quad 0 \leq t \leq b .
$$

Let $t_{*} \in[0, t] \subset[0, b]$ be such that $\mu(t)=\left|y\left(t_{*}\right)\right|$. By the previous inequality, we have for $t \in[0, b]$

$$
e^{-\omega t} E\left(\mu(t)^{2}\right) \leq 4 M E\left(|\phi(0)|^{2}+\beta\right)+2 M \int_{0}^{t} e^{-\omega s} p(s) \psi\left(E\left(\mu(s)^{2}\right)\right) d s
$$

Let us take the right-hand side of the above inequality as $v(t)$. Then we have

$$
\begin{gathered}
E\left(\mu(t)^{2}\right) \leq e^{\omega t} v(t) \quad \forall t \in[0, b], \\
c:=v(0)=4 M E\left(|\phi(0)|^{2}+\beta\right), \\
v^{\prime}(t)=2 M e^{-\omega t} p(t) \psi\left(E\left(\mu(t)^{2}\right)\right) \quad \text { a.e. } t \in[0, b] .
\end{gathered}
$$

Using the increasing character of $\psi$, we get

$$
v^{\prime}(t) \leq 2 M e^{-\omega t} p(t) \psi\left(e^{\omega t} v(t)\right) \quad \text { a.e. } t \in[0, b]
$$

We remark that

$$
\begin{aligned}
{\left[e^{\omega t} v(t)\right]^{\prime} } & =\omega e^{\omega t} v(t)+e^{\omega t} v^{\prime}(t) \\
& \leq|\omega| e^{\omega t} v(t)+2 M p(t) \psi\left(e^{\omega t} v(t)\right) \\
& \leq p_{*}(t)\left[e^{\omega t} v(t)+\psi\left(e^{\omega t} v(t)\right)\right]
\end{aligned}
$$

Thus

$$
\int_{v(0)}^{e^{\omega t} v(t)} \frac{d x}{x+\psi(x)} \leq \int_{0}^{b} p_{*}(s) d s<\int_{c}^{\infty} \frac{d x}{x+\psi(x)}
$$

From (H5), there exists a constant $K_{*}$ such that $e^{\omega t} v(t) \leq K_{*}, t \in[0, b]$, and there exists $M_{*}$ such that $\|y\|_{\widehat{M}_{2}} \leq M_{*}$. 
In the next steps, we will prove that $N$ is continuous and completely continuous. Step 1. $N$ is continuous.

Let $\left\{y_{n}\right\}$ be a sequence such that $y_{n} \rightarrow y$ in $\widehat{M}_{2}([-r, b], \overline{D(A)})$. Then for each $t \in[0, b]$,

$$
\begin{aligned}
& \left|N\left(y_{n}\right)(t)-N(y)(t)\right|^{2} \\
& \quad=\left|S^{\prime}(t)\left[h_{0}\left(y_{n}\right)-h_{0}(y)\right]+\frac{d}{d t} \int_{0}^{t} S(t-s)\left[f\left(s, y_{n s}\right)-f\left(s, y_{s}\right)\right] d w(s)\right|^{2} \\
& \quad \leq 2\left|S^{\prime}(t)\left[h_{0}\left(y_{n}\right)-h_{0}(y)\right]\right|^{2}+2\left|\frac{d}{d t} \int_{0}^{t} S(t-s)\left[f\left(s, y_{n_{s}}\right)-f\left(s, y_{s}\right)\right] d w(s)\right|^{2} \\
& \quad \leq 2 M e^{\omega t}\left|h_{0}\left(y_{n}\right)-h_{0}(y)\right|^{2}+2\left|\frac{d}{d t} \int_{0}^{t} S(t-s)\left[f\left(s, y_{n s}\right)-f\left(s, y_{s}\right)\right] d w(s)\right|^{2} \\
& \quad \leq 2 M e^{\omega t}\left|h_{0}\left(y_{n}\right)-h_{0}(y)\right|^{2}+2 M\left|\int_{0}^{t}\right| f\left(s, y_{n_{s}}\right)-f\left(s, y_{s}\right)|d w(s)|^{2} \\
& \quad \leq 2 M \max \left(e^{\omega b}, 1\right)\left|h_{0}\left(y_{n}\right)-h_{0}(y)\right|^{2}+2\left|\lim _{\lambda \rightarrow \infty} \int_{0}^{t} S^{\prime}(t-s) B_{\lambda} f\left(s, y_{n_{s}}\right)-f\left(s, y_{s}\right) d w(s)\right|^{2} .
\end{aligned}
$$

Then

$$
\begin{aligned}
E\left(\left|N\left(y_{n}\right)(t)-N(y)(t)\right|^{2}\right) \leq & E\left(2 M \max \left(e^{\omega b}, 1\right)\left|h_{0}\left(y_{n}\right)-h_{0}(y)\right|^{2}\right) \\
& +E\left(2\left|\lim _{\lambda \rightarrow \infty} \int_{0}^{t} S^{\prime}(t-s)\left[f\left(s, y_{n_{s}}\right)-f\left(s, y_{s}\right)\right] d w(s)\right|^{2}\right) \\
\leq & 2 M \max \left(e^{\omega b}, 1\right) E\left(\left|h_{0}\left(y_{n}\right)-h_{0}(y)\right|^{2}\right) \\
& +2 M \max \left(e^{|\omega| b}, 1\right) \int_{0}^{b} E\left(\left|f\left(s, y_{n_{s}}\right)-f\left(s, y_{s}\right)\right|^{2}\right) d s .
\end{aligned}
$$

Thus

$$
\begin{aligned}
& \left\|N\left(y_{n}\right)-N(y)\right\|_{\widehat{M}_{2}} \\
& \quad \leq \sqrt{2 M \max \left(e^{\omega b}, 1\right) b}\left|h_{0}\left(y_{n}\right)-h_{0}(y)\right| \\
& \quad+\sqrt{2 M b \max \left(e^{|\omega| b}, 1\right)}|| f\left(\cdot, y_{n}\right)-f(\cdot, y) \|_{\widehat{M}_{2}} \longrightarrow 0 \quad \text { as } n \longrightarrow \infty .
\end{aligned}
$$

Step 2. N maps bounded sets into bounded sets in $\widehat{M}_{2}([-r, b], H)$. 
8 Stochastic functional differential equations

Indeed, it is enough to show that there exists a positive constant $\ell$ such that for each $y \in \mathscr{B}_{q}=\left\{y \in \widehat{M}_{2}([-r, b], H):\|y\|_{\widehat{M}_{2}}^{2} \leq q\right\}$, one has $\|N(y)\|_{\widehat{M}_{2}} \leq \ell$.

Let $y \in B_{q}$, then for each $t \in[0, b]$, we have

$$
\begin{aligned}
|N(y)(t)|^{2}= & \left|S^{\prime}(t)\left[\phi(0)-h_{0}(y)\right]+\frac{d}{d t} \int_{0}^{t} S(t-s) f\left(s, y_{s}\right) d w(s)\right|^{2} \\
\leq & 2\left|S^{\prime}(t)\left[\phi(0)-h_{0}(y)\right]\right|^{2}+2\left|\frac{d}{d t} \int_{0}^{t} S(t-s) f\left(s, y_{s}\right) d w(s)\right|^{2} \\
\leq & 4 M \max \left(e^{\omega b}, 1\right)\left[\left|h_{0}(y)\right|^{2}+|\phi(0)|^{2}\right] \\
& +2\left|\lim _{\lambda \rightarrow \infty} \int_{0}^{t} S^{\prime}(t-s) B_{\lambda} f\left(s, y_{s}\right) d w(s)\right|^{2}
\end{aligned}
$$

Thus

$$
\begin{aligned}
\|N(y)\|_{\widehat{M}_{2}} \leq & 4 M \max \left(e^{\omega b}, 1\right) b\left[\left|h_{0}(y)\right|^{2}+|\phi(0)|^{2}\right] \\
& +2 M b \max \left(e^{|\omega| b}, 1\right)\left\|h_{q}\right\|_{L^{2}}:=\ell .
\end{aligned}
$$

Step 3. $N$ maps bounded sets into equicontinuous sets in $\widehat{M}_{2}([-r, b], H)$.

Let $t_{1}, t_{2} \in J, t_{1}<t_{2}, B_{q}$ be a bounded set of $\widehat{M}_{2}([-r, b], H)$ as in Step 2 and let $y \in B_{q}$. Then

$$
\begin{aligned}
\left|N(y)\left(t_{1}\right)-N(y)\left(t_{2}\right)\right|^{2}=\mid & \left.\mid S^{\prime}\left(t_{2}\right)-S^{\prime}\left(t_{1}\right)\right]\left[\phi(0)-h_{0}(y)\right]+\lim _{\lambda \rightarrow \infty} \int_{t_{1}}^{t_{2}} S^{\prime}\left(t_{2}-s\right) f\left(s, y_{s}\right) d w(s) \\
& +\left.\lim _{\lambda \rightarrow \infty} \int_{0}^{t_{1}}\left[S^{\prime}\left(t_{2}-s\right)-S^{\prime}\left(t_{1}-s\right)\right] f\left(s, y_{s}\right) d w(s)\right|^{2} \\
\leq & 4\left|S^{\prime}\left(t_{2}\right)-S^{\prime}\left(t_{1}\right)\right|^{2}\left[\left|h_{0}(y)\right|^{2}+|\phi(0)|^{2}\right] \\
& +4\left|\lim _{\lambda \rightarrow \infty} \int_{t_{1}}^{t_{2}} S^{\prime}\left(t_{2}-s\right) B_{\lambda} f\left(s, y_{s}\right) d w(s)\right|^{2} \\
& +4\left|\lim _{\lambda \rightarrow \infty} \int_{0}^{t_{1}}\left[S^{\prime}\left(t_{2}-s\right)-S^{\prime}\left(t_{1}-s\right)\right] B_{\lambda} f\left(s, y_{s}\right) d w(s)\right|^{2} .
\end{aligned}
$$


Hence

$$
\begin{aligned}
E\left(\left|N(y)\left(t_{1}\right)-N(y)\left(t_{2}\right)\right|^{2}\right) \leq & E\left(4\left|S^{\prime}\left(t_{2}\right)-S^{\prime}\left(t_{1}\right)\right|^{2}\left[\left|h_{0}(y)\right|^{2}+|\phi(0)|^{2}\right]\right) \\
& +E\left(4\left|\lim _{\lambda \rightarrow \infty} \int_{t_{1}}^{t_{2}} S^{\prime}\left(t_{2}-s\right) B_{\lambda} f\left(s, y_{s}\right) d w(s)\right|^{2}\right) \\
& +E\left(4\left|\lim _{\lambda \rightarrow \infty} \int_{0}^{t_{1}}\left[S^{\prime}\left(t_{2}-s\right)-S^{\prime}\left(t_{1}-s\right)\right] B_{\lambda} f\left(s, y_{s}\right) d w(s)\right|^{2}\right) \\
\leq & 4 b\left|S^{\prime}\left(t_{2}\right)-S^{\prime}\left(t_{1}\right)\right|^{2}\left[\left|h_{0}(y)\right|^{2}+|\phi(0)|^{2}\right] \\
& +4 b \int_{t_{1}}^{t_{2}}\left|S^{\prime}\left(t_{2}-s\right)\right|^{2} p(s) \psi(E(q)) d s \\
& +4 b \int_{0}^{t_{1}}\left|S^{\prime}\left(t_{2}-s\right)-S^{\prime}\left(t_{1}-s\right)\right|^{2} h_{q}(s) d s .
\end{aligned}
$$

The right-hand side tends to zero as $t_{2}-t_{1} \rightarrow 0$. Now we will show that $N \mathscr{S}_{q}(t)$ is relatively compact for every $t \in[0, b]$. In the case where $t=0$, we have $N \mathscr{B}_{q}(0)=\{\phi(0)-$ $\left.h_{0}(y)\right\}$ which is precompact from (H4). Let $0<t \leq b$ and $\epsilon<t \leq b$. For $y \in \mathscr{B}_{q}$,

$$
\begin{aligned}
N_{\epsilon}(y)(t)= & S^{\prime}(t)\left[\phi(0)-h_{0}(y)\right]+\lim _{\lambda \rightarrow \infty} \int_{t-\epsilon}^{t} S^{\prime}(t-s) B_{\lambda} f\left(s, y_{s}\right) d w(s) \\
& +\lim _{\lambda \rightarrow \infty} S^{\prime}(\epsilon) \int_{0}^{t-\epsilon} S^{\prime}(t-\epsilon-s) B_{\lambda} f\left(s, y_{s}\right) d w(s) .
\end{aligned}
$$

Since $S^{\prime}(t)$ is a compact operator, the set $H_{\epsilon}(t)=\left\{N_{\epsilon}(y)(t): y \in \mathscr{S}_{q}\right\}$ is precompact in $\overline{D(A)}$ for every $\epsilon, 0<\epsilon<t$. Moreover, for every $y \in \mathscr{B}_{q}$, we have

$$
\left|N_{\epsilon}(y)(t)-N(y)(t)\right|^{2} \leq\left|\lim _{\lambda \rightarrow \infty} \int_{t-\epsilon}^{t} S^{\prime}(t-s) B_{\lambda} f\left(s, y_{s}\right) d w(s)\right|^{2}
$$

Then

$$
\begin{aligned}
E\left(\left|N_{\epsilon}(y)(t)-N(y)(t)\right|^{2}\right) & \leq E\left(\left|\lim _{\lambda \rightarrow \infty} \int_{t-\epsilon}^{t} S^{\prime}(t-s) B_{\lambda} f\left(s, y_{s}\right) d w(s)\right|^{2}\right) \\
& \leq b \int_{t-\epsilon}^{t}\left\|S^{\prime}(t-s)\right\|_{B(H)}^{2} h_{q}(s) d s .
\end{aligned}
$$

Therefore, there are precompact sets arbitrarily close to the set $\left\{N_{\epsilon}(y)(t): y \in \mathscr{B}_{q}\right\}$. Hence the set $\left\{N_{\epsilon}(y)(t): y \in \mathscr{B}_{q}\right\}$ is precompact in $\overline{D(A)}$. 
The cases when $t_{1}, t_{2}<0$ or $t_{1}<0<t_{2}$ are obvious.

Set

$$
U=\left\{z \in \widehat{M}_{2}([-r, b], H):\|y\|_{\widehat{M}_{2}}<M_{*}+1\right\} .
$$

From the choice of $U$, there is no $y \in \partial U$ such that $y=\lambda N(y)$, for some $\lambda \in(0,1)$. As a consequence of the nonlinear alternative of Leray-Schauder type [7], we deduce that $N$ has a fixed point $y$ in $U$ which is an integral solution of the problem (1.1).

Remark 3.4. We can replace (H5) by the following condition.

$(\mathrm{H} 5)^{*}$ There exists a continuous nondecreasing function $\psi:[0, \infty) \rightarrow(0, \infty), p \in L^{1}([0$, $\left.b], \mathbb{R}_{+}\right)$, and nonnegative number $M_{*}>0$ such that

$$
\begin{gathered}
E\left(|f(t, u)|^{2}\right) \leq p(t) \psi\left(E\|u\|_{\widehat{M}_{2}}^{2}\right) \quad \text { for each } u \in \widehat{M}_{2}([-r, 0], H), \\
\frac{M_{*}}{4 M E\left(|\phi(0)|^{2}+\beta\right)+2 M \max \left(e^{|\omega| b}, 1\right) \psi\left(M_{*}\right) \int_{0}^{b} p(s) d s}>1
\end{gathered}
$$

Then the step on a priori estimates will be modified as follows.

Let $y$ be solution of the problem (1.1), then we have

$$
\begin{aligned}
|y(t)|^{2} & =\left|S^{\prime}(t)\left[\phi(0)-h_{0}(y)\right]+\frac{d}{d t} \int_{0}^{t} S(t-s) f\left(s, y_{s}\right) d w(s)\right|^{2} \\
& \leq 2\left|S^{\prime}(t)\left[\phi(0)-h_{0}(y)\right]\right|^{2}+2\left|\frac{d}{d t} \int_{0}^{t} S(t-s) f\left(s, y_{s}\right) d w(s)\right|^{2} \\
& \leq 4 M \max \left(e^{\omega b}, 1\right)\left[|\phi(0)|^{2}+\left|h_{0}(y)\right|^{2}\right] \\
& +2\left|\lim _{\lambda \rightarrow \infty} \int_{0}^{t} S^{\prime}(t-s) B_{\lambda} f\left(s, y_{s}\right)\right| d w(s)^{2} .
\end{aligned}
$$

Thus using $(\mathrm{H} 5)^{*}$, instead of (H5), we have

$$
\begin{aligned}
E\left(|y(t)|^{2}\right) \leq & E\left(4 M \max \left(e^{\omega b}, 1\right)\left[|\phi(0)|^{2}+\beta\right]\right) \\
& +E\left(2\left|\lim _{\lambda \rightarrow \infty} \int_{0}^{t} S^{\prime}(t-s) B_{\lambda} f\left(s, y_{s}\right) d w(s)\right|^{2}\right) \\
\leq & 4 M \max \left(e^{\omega b}, 1\right) E\left(|\phi(0)|^{2}+\beta\right) \\
& +2 M \max \left(e^{\omega b}, 1\right) b \int_{0}^{t} e^{-\omega s} E\left(\left|f\left(s, y_{s}\right)\right|^{2}\right) d s \\
\leq & 4 M \max \left(e^{\omega b}, 1\right) E\left(|\phi(0)|^{2}+\beta\right) \\
& +2 M \max \left(e^{\omega b}, 1\right) \int_{0}^{t} e^{-\omega s} p(s) \psi\left(E\left(\|\left. y_{s}\right|^{2}\right)\right) d s .
\end{aligned}
$$


We consider the function $\mu$ defined by

$$
\mu(t)=\sup \{|y(s)|: 0 \leq s \leq t\}, \quad 0 \leq t \leq b .
$$

Let $t_{*} \in[0, t] \subset[0, b]$ be such that $\mu(t)=\left|y\left(t_{*}\right)\right|$. By the previous inequality, we have for $t \in[0, b]$,

$$
\begin{aligned}
E\left(\mu^{2}(t)\right) \leq & 4 M \max \left(e^{|\omega| b}, 1\right) E\left(|\phi(0)|^{2}+\beta\right) \\
& +2 M \max \left(e^{|\omega| b}, 1\right) \int_{0}^{b} p(s) \psi\left(E\left(\mu^{2}(s)\right)\right) d s .
\end{aligned}
$$

Consequently,

$$
\frac{\|y\|_{\widehat{M}_{2}}}{4 M \max \left(e^{|\omega| b}, 1\right) E\left(|\phi(0)|^{2}+\beta\right)+2 M \psi\left(\|y\|_{\widehat{M}_{2}}\right)} \max \left(e^{|\omega| b}, 1\right) \int_{0}^{b} p(s) d s \leq 1 .
$$

Then by $(\mathrm{H} 5)^{*}$, there exists $M_{*}$ such that $\|y\|_{\widehat{M}_{2}} \neq M_{*}$.

Set

$$
U=\left\{y \in \widehat{M}_{2}([-r, b], \mathbb{R}):\|y\|_{\widehat{M}_{2}}<M_{*}\right\}
$$

and proceed as in Theorem 3.2.

\section{An example}

To apply the previous result, we consider the following partial stochastic differential equation:

$$
\begin{gathered}
\frac{\partial}{\partial t} u(t, x)=\Delta u(t, x)+f(t, u(t-r, x)) \frac{d w(t)}{d t}, \quad 0 \leq t \leq b, x \in \Omega, \\
u(t, x)=0, \quad 0 \leq t \leq b, x \in \partial \Omega, \\
u(t, x)+h_{t}(x)=v_{0}(x) \quad t \in[-r, 0], x \in \Omega,
\end{gathered}
$$

where $\Omega$ is a bounded open set of $\mathbb{R}^{n}$ with regular boundary $\partial \Omega, v_{0} \in C\left(\Omega, \mathbb{R}^{n}\right), f$ : $[0, b] \times \mathbb{R}^{n} \rightarrow \mathbb{R}^{n}$ is a given function, and $\triangle=\sum_{k=1}^{n}\left(\partial^{2} / \partial x_{k}^{2}\right)$. Consider $E=C(\bar{\Omega})$, the Banach space of continuous function on $\bar{\Omega}$ with values in $\mathbb{R}^{n}$. Define the linear operator $A$ on $E$ by

$$
A z=\triangle z \quad \text { in } D(A)=\{z \in C(\bar{\Omega}): z=0 \text { on } \partial \Omega, \triangle z \in C(\bar{\Omega})\} .
$$


Now, we have

$$
\overline{D(A)}=C_{0}(\bar{\Omega})=\{v \in C(\bar{\Omega}): v=0 \text { on } \partial \Omega\} \neq C(\bar{\Omega}) .
$$

It is well known from [5] that $A$ is sectorial, $(0,+\infty) \subseteq \rho(A)$, and for $\lambda>0$,

$$
|R(\lambda, \triangle)| \leq \frac{1}{\lambda}
$$

It follows that $A$ generates an integrated semigroup $(S(t))_{t \geq 0}$ and that $\left|S^{\prime}(t)\right| \leq e^{-\mu t}$ for $t \geq 0$ for some constant $\mu>0$. The partial stochastic differential equation (4.1) can be reformulated as the abstract semilinear stochastic differential equation (1.1) in $E$, where $F:[0, b] \times D(A) \rightarrow E$ is the Nemyskii operator given by

$$
F(t, u)(x)=f(t, u(t-r, x)) .
$$

If we assume that $f$ is an $L^{2}$-Carathéodory function satisfying (H5) and the conditions (H1), (H4) are satisfied, then the integral solution of (1.1) exists by Theorem 3.2.

\section{References}

[1] W. Arendt, Vector-valued Laplace transforms and Cauchy problems, Israel Journal of Mathematics 59 (1987), no. 3, 327-352.

[2] P. Balasubramaniam and S. K. Ntouyas, Global existence for semilinear stochastic delay evolution equations with nonlocal conditions, Soochow Journal of Mathematics 27 (2001), no. 3, 331-342.

[3] A. T. Bharucha-Reid, Random Integral Equations, Academic Press, New York, 1972.

[4] L. Byszewski, Theorems about the existence and uniqueness of solutions of a semilinear evolution nonlocal Cauchy problem, Journal of Mathematical Analysis and Applications 162 (1991), no. 2, 494-505.

[5] G. Da Prato and E. Sinestrari, Differential operators with nondense domain, Annali della Scuola Normale Superiore di Pisa. Classe di Scienze. Serie IV 14 (1987), no. 2, 285-344.

[6] G. Da Prato and J. Zabczyk, Stochastic Equations in Infinite Dimensions, Encyclopedia of Mathematics and Its Applications, vol. 44, Cambridge University Press, Cambridge, 1992.

[7] J. Dugundji and A. Granas, Fixed Point Theory. I, Monografie Matematyczne, vol. 61, Państwowe Wydawnictwo Naukowe (PWN), Warsaw, 1982.

[8] T. Hida, Analysis of Brownian Functionals, Carleton Mathematical Lecture Notes, no. 13, Carleton University, Ontario, 1975.

[9] T. Hida, H.-H. Kuo, J. Potthoff, and L. Streit, White Noise. An Infinite Dimensional Calculus, Mathematics and Its Applications, vol. 253, Kluwer Academic, Dordrecht, 1993.

[10] H. Kellerman and M. Hieber, Integrated semigroups, Journal of Functional Analysis 84 (1989), no. $1,160-180$.

[11] K. Liu, Carathéodory approximate solutions for a class of semilinear stochastic evolution equations with time delays, Journal of Mathematical Analysis and Applications 220 (1998), no. 1, 349-364.

[12] M. A. McKibben, Second-order damped functional stochastic evolution equations in Hilbert space, Dynamic Systems and Applications 12 (2003), no. 3-4, 467-487.

[13] Second-order neutral stochastic evolution equations with heredity, Journal of Applied Mathematics and Stochastic Analysis. JAMSA 2004 (2004), no. 2, 177-192.

[14] K. Sobczyk, Stochastic Differential Equations. With Applications to Physics and Engineering, Mathematics and Its Applications (East European Series), vol. 40, Kluwer Academic, Dordrecht, 1991. 
[15] T. Taniguchi, Successive approximations to solutions of stochastic differential equations, Journal of Differential Equations 96 (1992), no. 1, 152-169.

[16] C. P. Tsokos and W. J. Padgett, Random Integral Equations with Applications to Life Sciences and Engineering, Academic Press, New York, 1974.

M. Benchohra: Laboratoire de Mathématiques, Université Djillali Liabès - Sidi Bel Abbes, BP 89, 22000 Sidi Bel Abbes, Algeria

E-mail address: benchohra@univ-sba.dz

S. K. Ntouyas: Department of Mathematics, University of Ioannina, 45110 Ioannina, Greece E-mail address: sntouyas@cc.uoi.gr

A. Ouahab: Laboratoire de Mathématiques, Université Djillali Liabès - Sidi Bel Abbes, BP 89, 22000 Sidi Bel Abbes, Algeria

E-mail address: ouahab@univ-sba.dz 


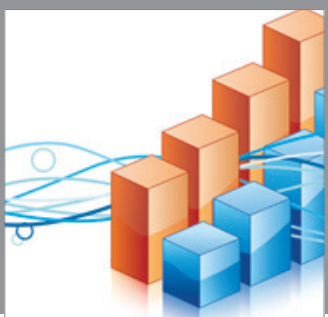

Advances in

Operations Research

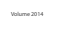

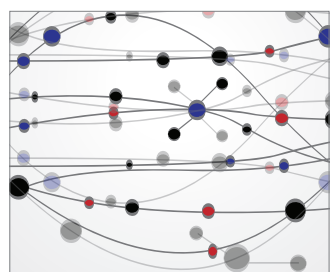

\section{The Scientific} World Journal
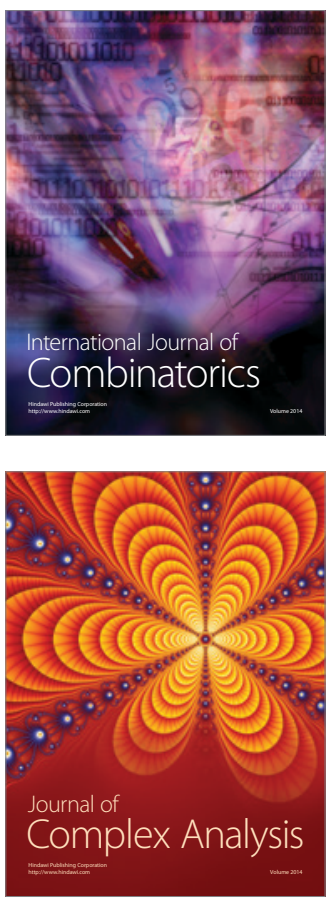

International Journal of

Mathematics and

Mathematical

Sciences
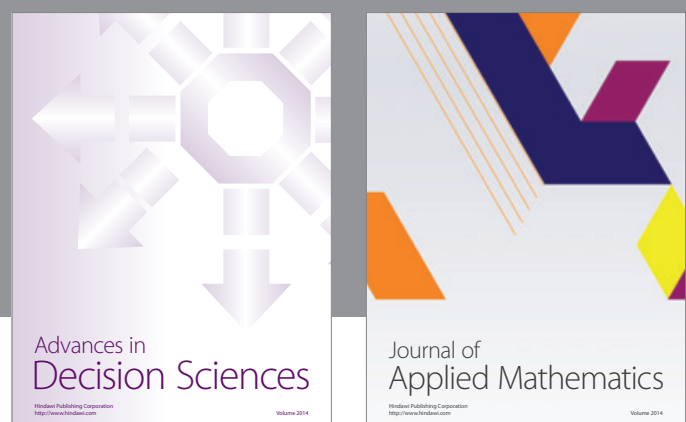

Journal of

Applied Mathematics
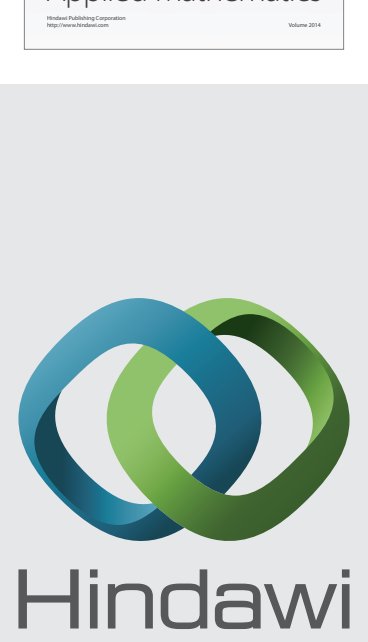

Submit your manuscripts at http://www.hindawi.com
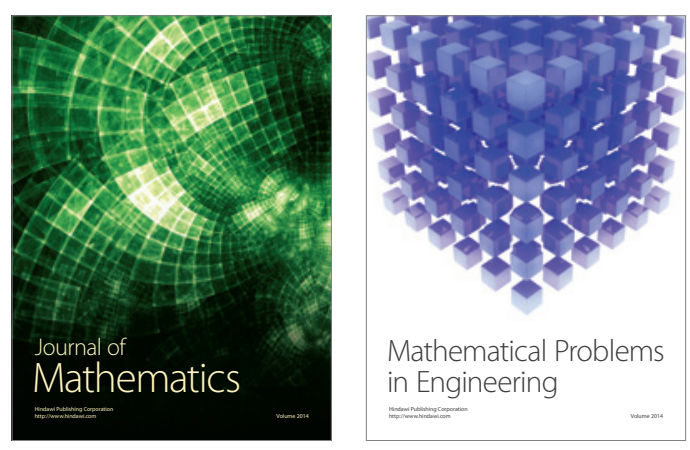

Mathematical Problems in Engineering
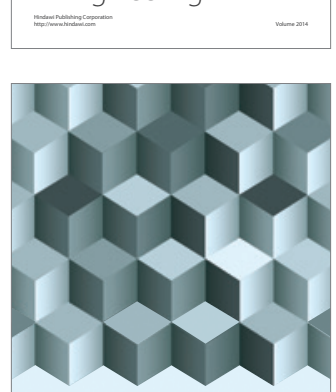

Journal of

Function Spaces
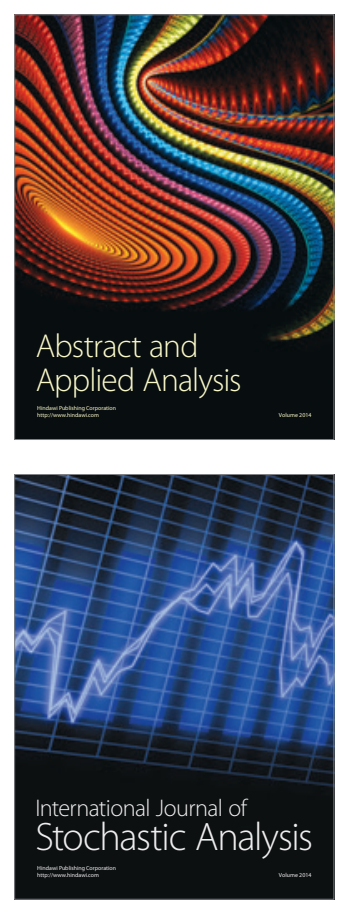

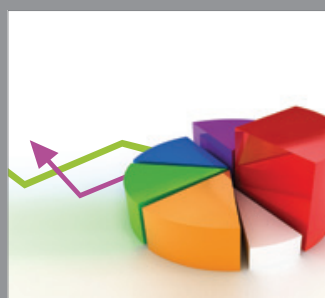

ournal of

Probability and Statistics

Promensencen
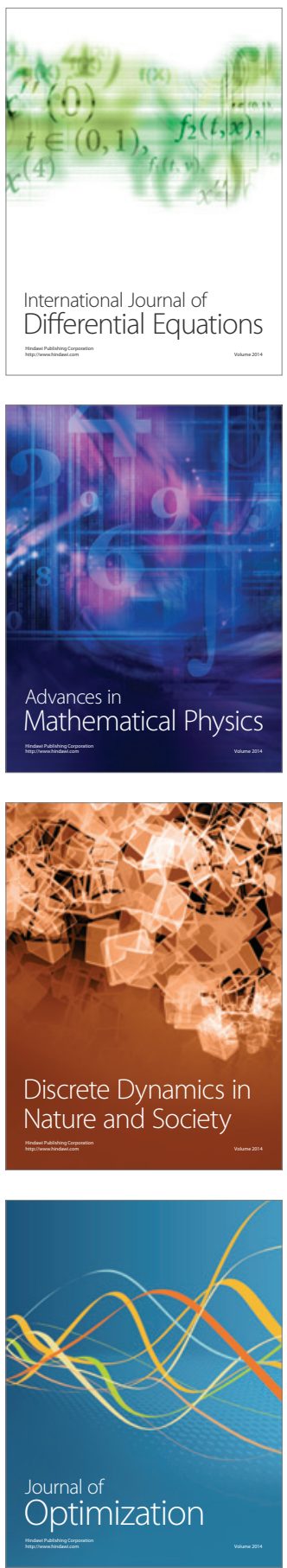